\title{
EDITORIAL
}

\section{Diagnostic criteria for the sleep apnoea syndrome: time for consensus?}

\author{
W.T. McNicholas*
}

The traditional diagnosis of sleep apnoea syndrome has been based on a particular apnoea frequency during sleep, and was originally set at $\geq 5$ apnoeas $\cdot \mathrm{h}^{-1}$ of sleep during the 1970s [1]. However, as experience developed with the clinical spectrum of patients with sleep apnoea, it was realized that hypopnoeas during sleep could have a similar clinical significance as complete apnoeas [2], and this realization led to the present clinical diagnostic criteria, which are based on the combined frequency of apnoeas and hypopnoeas during sleep. Unfortunately, there is not complete agreement as to what apnoea and hypopnoea frequency represents a clinically significant sleep apnoea syndrome, with some centres using a level of 10 episodes $\cdot h^{-1}$ [3-5], and others 15 episodes $\cdot h^{-1}[6,7]$.

A number of obvious deficiencies can be identified in the present approach to the diagnosis of obstructive sleep apnoea (OSA). Firstly, not all patients with an apnoea/hypopnoea index (AHI) of greater than 10 or 15 episodes $\cdot \mathrm{h}^{-1}$ of sleep appear to have a clinically significant OSA syndrome. It has long been recognized that apnoeas during sleep become more frequent with advancing age, and many apparently normal elderly people have an AHI greater than the above thresholds [8,9]. It is now accepted that a diagnosis of clinically significant OSA should be accompanied by compatible symptoms, and not be based simply on an arbitrary AHI threshold, particularly in borderline cases. Clinically significant OSA can occur, particularly among younger patients, at AHI frequencies below 10 episodes $\cdot h^{-1}$, and the recent description of the upper airway resistance syndrome [10] indicates that episodic partial upper airway obstruction without significant hypopnoea can mimic OSA in most respects, and can respond to traditional treatment modalities, such as nasal continuous positive airway pressure (nCPAP). However, on the other hand, population surveys among otherwise apparently normal elderly individuals have shown that those with apnoea frequencies above the traditional diagnostic threshold have reduced mental performance compared with those individuals without significant apnoea frequencies, which casts some doubt on the apparent normality of those individuals with a high apnoea frequency [8]. These issues indicate that considerable work remains to be done to clarify the clinical and laboratory criteria for a significant OSA syndrome.

The realization that compatible clinical features are an important part of the diagnosis of OSA has led a number of centres to seek clinical features that are predictive of the presence of OSA. However, in most studies,

*Dept of Respiratory Medicine and the Respiratory Sleep Laboratory, St. Vincent's Hospital, Dublin 4, Ireland. individual clinical features have not been found helpful in predicting OSA. They may increase the suspicion of OSA being present but are of little value in excluding OSA. Certain symptoms, such as snoring and excessive daytime sleepiness, have a high positive predictive value, but also have a relatively low negative predictive value, which implies that the presence of such clinical features may increase the suspicion of OSA, but their absence cannot be used to exclude OSA with any degree of certainty. Other clinical features have been reported to be more specific, particularly witnessed apnoeas [6], a tendency to doze at the wheel while driving [11], and neck circumference [12]. Not surprisingly, several reports have demonstrated that when clinical features are combined, the diagnostic value is improved, and the further addition of simple overnight oximetry further improves diagnostic accuracy. However, we have recently found, in a prospective study [13], that the combination of clinical features with simple oximetry allows a correct prediction of the presence or absence of OSA in only one third of patients. The remaining two thirds of patients would require more detailed sleep studies to allow a correct diagnosis.

A second major problem with the present diagnostic criteria for OSA is that since the formal diagnosis is based on a particular apnoea/hypopnoea frequency during sleep, these criteria necessitate the measurement of both sleep and respiration, which essentially implies the need for full polysomnographic sleep studies. Since recent epidemiological studies indicate a prevalence for clinically significant OSA of approximately one percent of the male population in Europe [14], and about $4 \%$ in North America [15], the resources required to assess such numbers by full sleep studies in sleep laboratories would be immense. The development of large numbers of limited diagnostic systems in recent years represents a recognition of these logistic problems. Such systems measure a number of different variables, without formal assessment of sleep stage. Unfortunately, there is no uniformity among these devices, and the only consistent variable common to all such systems is oxygen saturation $\left(\mathrm{Sa}_{\mathrm{a}} \mathrm{O}_{2}\right)$. Most offer some measure of respiration, based either on nasal flow or chest bands. Other variables measured in some systems include body motion, video monitoring, heart rhythm, and/or obstructed breathing efforts.

The value of performing overnight oximetry alone in the assessment of patients with suspected OSA has been examined previously, using observer pattern recognition $[16,17]$. Whilst oximetry provides a very characteristic pattern in very severe cases of OSA, it is much less helpful in milder cases of the disease, and cannot confidently 
exclude the diagnosis. We have found that using specific measurements, namely total number of dips $\geq 4 \%$ and nadir of $\mathrm{Sa}_{\mathrm{a}} \mathrm{O}_{2}$, only about one third of patients can be correctly designated as OSA or non-OSA [13]. Thus, whilst oximetry demonstrates a typical pattern in severe cases of OSA which cannot be mistaken for any other condition, it cannot be used alone in the majority of patients to diagnose the presence or absence of OSA, and other additional measurements are required.

Many of the limited diagnostic systems presently on the market have been poorly validated, and there is a major need for properly designed, independently controlled validation studies to compare the accuracy and reproducibility of these limited systems against the present gold standard, namely polysomnography. There is also a great need for an authoritative international consensus on the basic parameters needed to diagnose the presence or absence of clinically significant OSA in the clinical setting. Such a consensus would be of major assistance to the manufacturers of diagnostic equipment by laying out broad parameters along which these limited systems should be developed. However, it must be recognized that the technology in this area is rapidly changing and, therefore, this consensus would require regular review and revision. A previous European consensus, sponsored by the European Commission, reported on this subject in 1992 [18], but the technological advances since then require a major review. Since most diagnostic systems in the field of sleep disorders target a worldwide distribution, a joint consensus incorporating at least European and North American views appears desirable.

The growing numbers of patients presenting for assessment of possible OSA have swamped many of the existing clinical sleep laboratories, and increasing attention is being focused on the possibility of performing domiciliary sleep studies. Many portable systems are now available, some of which provide facilities for full polysomnography, whilst others allow limited diagnostic studies. While there are obvious advantages of such home-based studies, particularly improved sleep quality and cost-savings, there are also disadvantages. The lack of technician supervision of the study means that leads which become dislodged are not replaced during the study, and the likelihood of technically unsatisfactory studies is therefore higher. A cost-benefit analysis of such home-based studies is required, which takes into account the above considerations.

In view of these considerations, there is a pressing need for an International Consensus on the clinical assessment of patients with suspected sleep apnoea, representing the views of the major scientific bodies that are active in the field, including the European Respiratory Society. Such a Consensus should address two major questions: 1) What are the most appropriate diagnostic criteria to allow an accurate diagnosis of OSA? and 2) What are the minimum physiological variables necessary to allow a confident assessment of the presence or absence of OSA?

The prevalence of OSA is now understood to be so great that the clinical assessment of these patients will be likely to involve clinicians outside major sleep centres, who may not have as detailed an understanding of the syndrome as clinicians who have undertaken specific training in sleep medicine. It is important, therefore, that clear-cut guidelines and criteria be established for the assessment and management of patients with suspected OSA.

\section{References}

1. Guilleminault C, Tilkian A, Dement WC. The sleep apnea syndromes. Annu Rev Med 1976; 27: 465-484.

2. Gould GA, Whyte KF, Rhind GB, et al. The sleep hypopnea syndrome. Am Rev Respir Dis 1988; 137: 895-898.

3. Viner S, Szalai JP, Hoffstein V. Are history and physical examination a good screening test for sleep apnea? Ann Intern Med 1991; 115: 356-359.

4. Bliwise DL, Nekich JC, Dement WC. Relative validity of self-reported snoring as a symptom of sleep apnea in a sleep clinic population. Chest 1991; 99: 600-608.

5. Flemons WW, Whitelaw WW, Brant R, Remmers JE. Likelihood ratios for a sleep apnea clinical prediction rule. Am J Respir Crit Care Med 1994; 150: 1279-1285.

6. Crocker BD, Olson LG, Saunders NA, et al. Estimation of the probability of disturbed breathing during sleep before a sleep study. Am Rev Respir Dis 1990; 142: 14-18.

7. Douglas NJ, Thomas S, Jan MA. Clinical value of polysomnography. Lancet 1992; 339: 347-350.

8. Ancoli-Israel S, Kripke DF, Mason W, Kaplan OJ. Sleep apnea and periodic movements in an aging sample. $J$ Gerontol 1985; 40: 419-425.

9. Moldofsky H, Goldstein R, McNicholas WT, Lue F, Zamel N, Phillipson EA. Disordered breathing during sleep and overnight intellectual deterioration in patients with pathological ageing. In: Guilleminault C, Lugaresi E, eds. Sleep/Wake Disorders: Natural History, Epidemiology and Long-term Evolution. New York, Raven Press, 1983; pp. 143-150.

10. Guilleminault C, Stoohs R, Clerk A, Cetel M, Maistros P. A cause of excessive daytime sleepiness: the upper airway resistance syndrome. Chest 1993; 104: 781-787.

11. Kump K, Whalen C, Tishler PV, et al. Assessment of the validity and utility of a sleep-symptom questionnaire. Am J Respir Crit Care Med 1994; 150: 735-741.

12. Katz I, Stradling J, Slutsky AS, Zamel N, Hoffstein V. Do patients with obstructive sleep apnea have thick necks? Am Rev Respir Dis 1990; 141: 1228-1231.

13. Deegan PC, McNicholas WT. Predictive value of clinical features for the obstructive sleep apnoea syndrome. Eur Respir J 1996; 9: 117-124.

14. Stradling JR, Crosby JH. Predictors and prevalence of obstructive sleep apnoea and snoring in 1,001 middleaged men. Thorax 1991; 46: 85-90.

15. Young T, Palta M, Dempsey J, Skatrud J, Weber S, Badr $\mathrm{S}$. The occurrence of sleep-disordered breathing among middle-aged adults. N Engl J Med 1993; 328: 1230-1235.

16. Stradling J, Apps M, Calverley P, Chadwick G, McNicholas WT. Adequacy of oximetry-alone studies for the diagnosis of sleep and breathing disorders. J Ambul Monit 1989; 2: 197-201.

17. Cooper BG, Veale D, Griffiths CJ, Gibson GJ. Value of nocturnal oxygen saturation as a screening test for sleep apnoea. Thorax 1991; 46: 586-588.

18. Stradling JR, McNicholas WT, and the Pulmonary Medicine subgroup of the European Community concerted action on methodology for the analysis of the Sleep-Wakefulness continuum. Sleep studies for sleep-related breathing disorders. A Consensus Report. J Sleep Res 1992; 1: 265-273. 